Related content

\section{Dynamical correlations in quasi-one-dimensional electron gas}

To cite this article: C. Bulutay and B. Tanatar 1998 EPL 43572

View the article online for updates and enhancements. $\frac{\text { Collective excitations and instabilities in }}{\text { double-wire electron - hole systems }}$ $\mathrm{N}$ Mutluay and B Tanatar
- Correlations in two-dimensional electron gas: Random-phase approximation with exchange and ladder results
F. Pederiva, E. Lipparini and $\mathrm{K}$.
Takayanagi
Exchange - correlation effects in semiconductor double-quantum-wire systems
N Mutluay and B Tanatar

\section{Recent citations}

- Liquid 4 He at Zero Temperature and the
STLS Scheme
A. Doroudi
- Dynamic correlation effects on the
plasmon dispersion in a two-dimensional
electron gas
V. Moldoveanu et al
- Search of Self-Organized Criticality
$\frac{\text { Processes in Magnetically Confined }}{\text { Plasmas: Hints from the Reversed Field }}$
Pinch Configuration
G. Serianni et al




\title{
Dynamical correlations in quasi-one-dimensional electron gas
}

\author{
C. Bulutay ${ }^{1}$ and B. Tanatar ${ }^{2}$ \\ ${ }^{1}$ Department of Electrical and Computer Engineering, University of California \\ Santa Barbara, California, 93106, USA \\ Department of Electrical and Electronics Engineering \\ Middle East Technical University - 06531 Ankara, Turkey \\ ${ }^{2}$ Department of Physics, Bilkent University - Bilkent, 06533 Ankara, Turkey
}

(received 6 April 1998; accepted in final form 13 July 1998)

PACS. 71.10Ca - Electron gas, Fermi gas.

PACS. 71.45Gm- Exchange, correlation, dielectric and magnetic functions, plasmons.

PACS. 73.20Dx - Electron states in low-dimensional structures (superlattices, quantum well structures and multilayers).

\begin{abstract}
We study the short-range correlations in a quasi-one-dimensional electron gas within the dynamical version of the self-consistent field theory. The static structure factor exhibits a peak structure at low densities. The zero-frequency limit of the dynamic local-field factor has structure not encountered in the previous applications of the present method. The large oscillations at low densities observed in the pair-correlation function indicates a transition to a partially ordered state.
\end{abstract}

The many-body problem of a system of electrons interacting via the long-range Coulomb forces comprises a useful model to study a number of physical systems such as metals and semiconductor heterojunctions. The recent advances in the fabrication techniques have made it possible to produce effectively two- and quasi-one-dimensional (Q1D) electronic systems, which in turn have stimulated a surge of theoretical activity exploring their ground state properties. The exchange-correlation effects describing the screening properties of charge carriers in low-dimensional systems are nowadays starting to become of experimental relevance [1].

The dynamical nature of the correlations in interacting electron systems is gaining attention in recent years. Moudgil et al. [2] have studied the dynamic density and spin-density correlations in a two-dimensional (2D) electron fluid within the Singwi-Tosi-Land-Sjölander (STLS) [3] theory. Richardson and Ashcroft [4] have evaluated the wave vector and frequencydependent local-field factors for a 3D electron gas using a variational approach to solve the integral equations for the response functions. Takayanagi and Lipparini [5] have solved Dyson's equation in the particle-hole channel to determine the effective interaction between electrons, and calculated the dynamic dielectric function. The importance of dynamical local fields is evident in various applications such as the construction of effective electron-electron interactions [4] which are relevant for Coulomb interaction induced superconductivity, determining the plasmon lifetime, and setting up the exchange-correlation potential in the context of time-dependent density-functional theory [6].

(C) EDP Sciences 
The STLS approximation provides an improvement over the random-phase approximation (RPA) which neglects correlation effects. The local fields take the repulsion hole around an electron into account to describe the correlation effects. Reasonable estimates of the ground state properties of various electronic systems are obtained within the STLS, and its physically appealing structure makes it a useful tool in studying the dielectric properties of charged quantum systems. The Q1D electron gas was investigated by a number of researchers $[7,8]$ within the static STLS approach which treats the local-field factor as a frequency-independent quantity. The importance of short-range correlation effects in Q1D electron systems is also beginning to be recognized [9]. The dynamic (or quantum) version of the STLS scheme makes use of the quantum-mechanical equation of motion for the Wigner distribution function [10]. The main finding of the previous applications has been that the quantum effects incorporated in $G(q, \omega)$ influence significantly the short-range correlations.

The purpose of this paper is to present our results on the dynamical correlations in a Q1D electron gas. We employ the dynamical version of the STLS approximation to solve selfconsistently for the static structure factor $S(q)$ and the wave vector and frequency dependent local-field factor $G(q, \omega)$ for a range of densities. We find that the dynamical correlations become very important at low densities, and the resulting ground state properties are quite different than those evaluated within the static approximations. In the following we outline our calculational method and discuss our results.

We model the Q1D electron system as obtained from the zero-thickness 2D electron gas under a confining potential [11]. This yields $V(q)=\left(e^{2} / \epsilon_{0}\right) e^{x} K_{0}(x)$ for the Coulomb interaction between the electrons assumed to be in the lowest subband. Here $x=(b q / 2)^{2}$, where $b$ is the lateral width of the quantum wire determined by the confining oscillator frequency, and $\epsilon_{0}$ is the background dielectric constant. The system is characterized by the dimensionless density parameter $r_{\mathrm{s}}=a / a_{\mathrm{B}}^{*}$, where $a$ is the average interparticle distance (i.e. $n=1 / 2 a$ in terms of the linear number density $n$ ), and $a_{\mathrm{B}}^{*}=\epsilon_{0} /\left(m^{*} e^{2}\right)$ is the effective Bohr radius (we take $\hbar=1$ ). The single-subband approximation, which implies that the Fermi energy remains smaller than the intersubband energy difference, is justified for $r_{\mathrm{s}}>\pi / 2^{5 / 2}\left(b / a_{\mathrm{B}}^{*}\right)$. We calculate the correlation effects employing the self-consistent field approximation of Singwi et al. [3] generalized to include dynamic correlations [10] which introduces a local-field correction to the bare interaction $V(q)$ arising from the short-range correlations between the electrons. In this work, we use the Fermi-liquid description of the Q1D electron gas in contrast to the Luttinger-liquid model. It has been argued [12] that the impurity scattering and finite-temperature effects would restore the quasi-particle picture in these systems. We simulate the effects of disorder by using a phenomenological broadening in the density-density response function that enters the formalism.

The density-density response function in the STLS theory is given in the form of a generalized RPA,

$$
\chi(q, \omega)=\frac{\chi_{0}(q, \omega)}{1-V_{\mathrm{eff}}(q, \omega) \chi_{0}(q, \omega)},
$$

where $\chi_{0}(q, \omega)$ is the zero-temperature dynamic susceptibility of a noninteracting electron gas,

$$
\chi_{0}(q, i \omega)=\frac{m}{2 \pi q} \ln \left|\frac{\omega^{2}+\omega_{-}^{2}}{\omega^{2}+\omega_{+}^{2}}\right|
$$

where $\omega_{ \pm}=\left|q^{2} / 2 m \pm q k_{\mathrm{F}} / m\right|$ are the boundaries for particle-hole excitations. Here we have evaluated $\chi_{0}$ on the imaginary frequency axis to facilitate the subsequent calculations. 


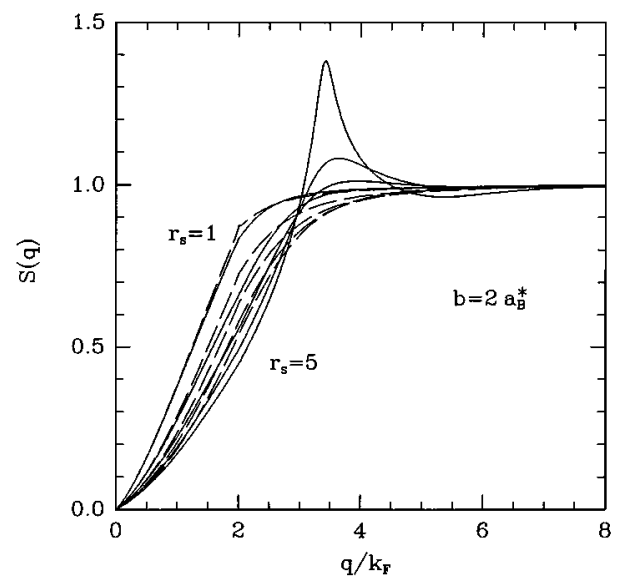

Fig. 1

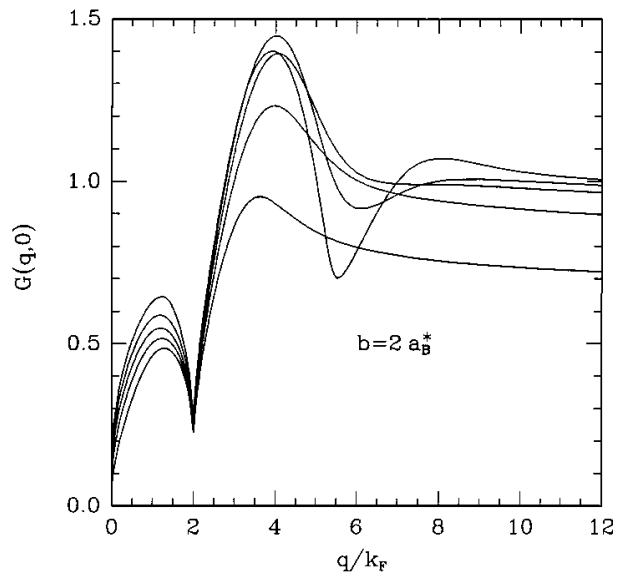

Fig. 2

Fig. 1. - The static structure factor $S(q)$ within the dynamic (solid lines) and static (dashed lines) STLS at $r_{\mathrm{s}}=1-5$, for a quantum wire of width $b=2 a_{\mathrm{B}}^{*}$.

Fig. 2. - The zero-frequency local-field factor $G(q, 0)$ calculated within the dynamic STLS for $r_{\mathrm{s}}=1-5$, and $b=2 a_{\mathrm{B}}^{*}$.

The static structure factor is related to the density-density response function through the fluctuation-dissipation theorem

$$
S(q)=-\frac{1}{n \pi} \int_{0}^{\infty} \mathrm{d} \omega \chi(q, i \omega)
$$

where we have used the analytic continuation of the response function to the complex frequency plane followed by the Wick rotation of the frequency integral. Since the plasmon excitations dominate over the particle-hole pair excitations in one-dimensional electron systems, the above procedure becomes very effective for numerical computations. The effective interaction $V_{\text {eff }}(q, \omega)=V(q)[1-G(q, \omega)]$ is defined in terms of the frequency-dependent (dynamic) localfield factor

$$
G(q, \omega)=-\frac{1}{n} \int_{-\infty}^{\infty} \frac{\mathrm{d} q^{\prime}}{2 \pi} \frac{\chi_{0}\left(q, q^{\prime} ; \omega\right)}{\chi_{0}(q, \omega)} \frac{V\left(q^{\prime}\right)}{V(q)}\left[S\left(q-q^{\prime}\right)-1\right],
$$

where the inhomogeneous free-electron response function, defined by

$$
\chi_{0}\left(q, q^{\prime} ; \omega\right)=2 \int_{-\infty}^{\infty} \frac{\mathrm{d} p}{2 \pi} \frac{f\left(p+q^{\prime} / 2\right)-f\left(p-q^{\prime} / 2\right)}{\omega-p q / m+i \eta},
$$

is used, with $f(q)$ being the distribution function for noninteracting electrons. Note that for $q^{\prime}=q$, the inhomogeneous response function reduces to the familiar homogeneous one $\chi_{0}(q, \omega)$. The derivation leading to the frequency-dependent local-field factor $G(q, \omega)$ is similar to that in the static STLS approximation. The hierarchy of coupled equations satisfied by the Wigner distribution functions is truncated with the assumption that the two-particle Wigner distribution function may be written as a product of one-particle distribution functions and the pair-correlation function [10].

We have solved the self-consistent equations for the static structure factor $S(q)$ and the dynamic local-field correction evaluated on the imaginary frequency axis $G(q, i \omega)$ set out 
in the previous paragraphs. The calculated $S(q)$ along with the results of the static STLS approximation are depicted in fig. 1 . We observe a certain similarity between the static and dynamic STLS results at small $r_{\mathrm{s}}$ (i.e. $\left.r_{\mathrm{s}} \lesssim 1\right)$. As $r_{\mathrm{s}}$ increases, $S(q)$ calculated within the dynamic STLS becomes vastly different from the static STLS result. Most notably, for $r_{\mathrm{s}} \geq 3, S(q)$ exhibits a broad peak around $q \approx 3.5 k_{\mathrm{F}}$, which increases in magnitude. The wave vector at which $S(q)$ is peaked is close to the first star of the reciprocal lattice of a one-dimensional chain which signals the evolution of an ordered structure. Such a behavior of $S(q)$ is reminiscent of the results of Quantum Monte Carlo (QMC) simulations [13] and highly sophisticated integral equation based theories [14] at very low densities. The static STLS theories $[7,8]$, as well as the ladder approximation calculations [9], do not produce an $S(q)$ with a marked peak for the same densities.

The dynamic local-field factor evaluated self-consistently on the imaginary frequency axis has a smooth and uninteresting dependence on frequency. Similar results for $G(q, i \omega)$ were obtained in a 3D electron gas within the ladder approximation scheme of Pederiva et al. [15] The real and imaginary parts of $G(q, \omega)$ may be obtained from $G(q, i \omega)$ by the analytic continuation $i \omega \rightarrow \omega+i \eta$. We found that the real and imaginary parts of $G(q, \omega)$ are oscillating functions of $\omega$ as in the 2D case [2]. We defer the detailed study of the effects of the frequency-dependent $G(q, \omega)$ on the dynamical properties such as the plasmon dispersion and energy-loss function for future work, and concentrate on the static properties. In fig. 2, we show the zero-frequency sector of our self-consistently calculated local-field factor $G(q, 0)$, at several densities. $G(q, 0)$ for a Q1D electron gas exhibits peculiar features not encountered in $2 \mathrm{D}$ and $3 \mathrm{D}$ systems. Firstly, there is a sharp dip at $q=2 k_{\mathrm{F}}$ and a small maximum around $q \approx k_{\mathrm{F}}$. At around $q \approx 4 k_{\mathrm{F}}$ there is another maximum with magnitude exceeding unity. The interesting features observed at $4 k_{\mathrm{F}}$ and possibly at $6 k_{\mathrm{F}}$ are suggestive in associating them with instabilities stemming from multi-particle effects [16]. Even though the dynamic STLS theory involves the dynamics of only the single particle-hole pair excitations, the structure in

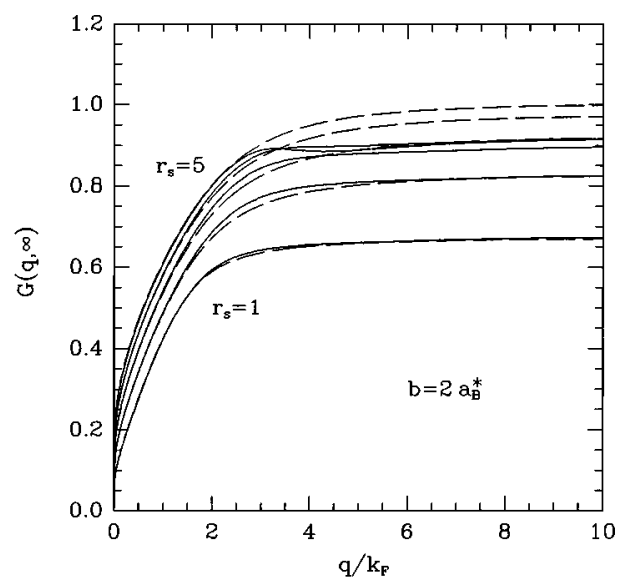

Fig. 3

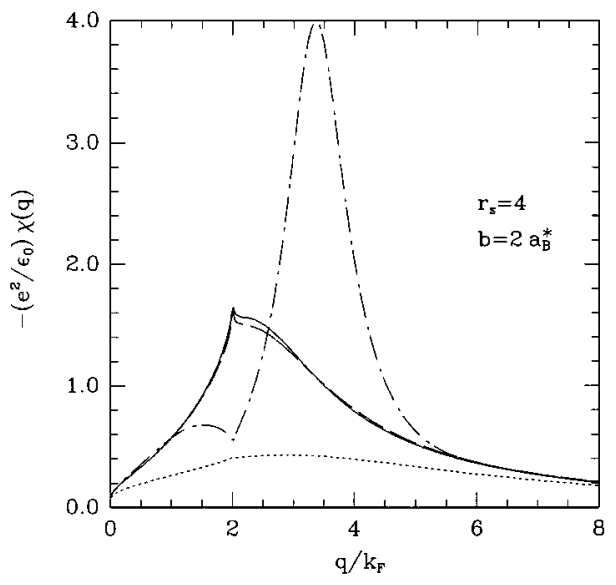

Fig. 4

Fig. 3. - The large frequency limit of the dynamic local-field factor $G(q, \infty)$ (solid lines) and the static local-field factor $G(q)$ (dashed lines) for $r_{\mathrm{s}}=1-5$, and $b=2 a_{\mathrm{B}}^{*}$.

Fig. 4. - The static density-density response function for a quantum wire of $b=2 a_{\mathrm{B}}^{*}$ at $r_{\mathrm{s}}=4$. The dotted line shows the RPA result. In the curves indicated by dashed, solid, and dash-dotted lines static STLS $G(q), G(q, \infty)$, and $G(q, 0)$ are used, respectively. 


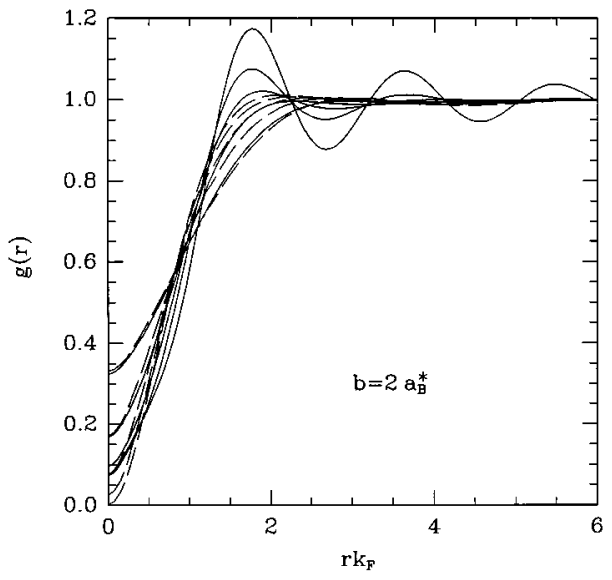

Fig. 5. - The pair distribution function $g(r)$ within the dynamic (solid lines) and static (dashed lines) STLS for $r_{\mathrm{s}}=1-5$, and $b=2 a_{\mathrm{B}}^{*}$.

$G(q, 0)$ may point to the importance of multi-pair excitations. In the earlier calculations of $G(q, 0)$ for 3D electron systems [10], it has been argued that when $G(q, 0)$ exceeds unity, the system becomes unstable towards the formation of a charge density wave (CDW). The QMC simulations [13] have studied the uniform fluid phases but a CDW state was not explored. Our calculations (using the procedure of ref. [10]) indicate a CDW instability for $r_{\mathrm{s}} \gtrsim 3$ at $q \approx 3 k_{\mathrm{F}}$ for a quantum wire of $b=2 a_{\mathrm{B}}^{*}$.

As in the 3D case [10], one can show that for fixed $q$ and large $\omega$, the local-field factor behaves as $\lim _{\omega \rightarrow \infty} G(q, \omega)=G(q)+\mathcal{O}\left(\omega^{-2}\right)$, where $G(q)$ is the result of the static STLS. We observe in fig. 3 that the high-frequency limit of the local-field factor $G(q, \infty)$ approximates the static $G(q)$ better than $G(q, 0)$. In particular, at small $r_{\mathrm{s}}, G(q, \infty)$ and $G(q)$ are very similar, and their long-wavelength limits coincide at all densities. At large $q$, because of the dynamical correlations $G(q, \infty)$ and $G(q)$ of the static STLS show notable differences which may be attributed to the contribution of $\mathcal{O}\left(\omega^{-2}\right)$ corrections. Although $G(q)$ is a monotone increasing function of $q$, approaching unity as $q \rightarrow \infty, G(q, \infty)$ starts to develop a mild peak around $q \approx 3 k_{\mathrm{F}}$ for large $r_{\mathrm{s}}$, and the difference between the large- $q$ limits of $G(q, \infty)$ and the static $G(q)$ becomes more noticeable. To further attest the differences between the various local-field corrections, we calculate the static density-density response function $\chi(q)$. Figure 4 shows our results when different local-field factors are used in comparison to the RPA result. The static STLS result $G(q)$ and the dynamically calculated high-frequency result $G(q, \infty)$ yield similar $\chi(q)$. However, the dynamically calculated zero-frequency local-field $G(q, 0)$ produces a small dip at $q=2 k_{\mathrm{F}}$, and a large peak around $q \approx 3.4 k_{\mathrm{F}}$. The magnitude of the peak in $\chi(q)$ increases with increasing $r_{\mathrm{s}}$. This is similar to the CDW instability developing in 2D electron systems at low density studied by Świerkowski et al. [17].

Finally, we show the dynamic and static STLS pair-correlation functions $g(r)$ (which are obtained by Fourier-transforming the $S(q)$ 's) in fig. 5 for $r_{\mathrm{s}}=1-5$. For the densities of interest, $g(0)$ remains positive. With increasing $r_{\mathrm{s}}, g(r)$ exhibits more pronounced oscillatory behavior. Such oscillations, typically seen in QMC calculations, indicate partial localization of the charged particles. The RPA and static STLS approaches are not capable of capturing such a localized state. Our numerical results may be interpreted as signaling the transition to the Wigner crystal phase. QMC simulations and hypernetted-chain-type methods could be used to explore and test the behavior here, particularly the competition between a CDW state 
and a Wigner crystal. It might also be useful to employ our liquid-structure data as input in density-functional-type theories [18] to predict the Wigner crystallization density in this system.

The major shortcoming of the static and dynamic STLS theories (in the present context) is that the exact large- $q$ behavior [19] of the local-field factor is not reproduced. On the one hand, the agreement between the structure factors and ground-state energies calculated in the dynamic STLS and QMC approaches [13] is reasonable for not too low densities. On the other hand, the QMC simulations of the response functions [20] in two- and three-dimensional electron gas indicate that the static local field factor is an increasing function of $q$ in contrast to the constant value attained in STLS approximations. The results of our dynamical STLS calculations should be useful in elucidating the differences between $G(q, 0)$ and $G(q, \infty)$ and in understanding the dynamical aspects of the short-range correlation effects in Q1D structures. To explore the latter point, more detailed studies on the dynamical properties using the frequency-dependent $G(q, \omega)$ are required.

Although we have used a specific model for the Coulomb interactions in a quantum wire, we believe that our calculations are representative of Q1D electron systems in general. We have also performed calculations for different quantum wire widths and found that the $r_{\mathrm{s}}$ value around which the correlation effects drive the system towards a localized state increases with the width parameter $b$.

In summary, we have calculated the static structure factor and dynamical local-field corrections for a Q1D electron system within the dynamic STLS approximation. Our results are vastly different than the static version of the STLS theory. The peak structure in $S(q)$ for large $r_{\mathrm{s}}$, and the oscillatory behavior of the pair-correlation function $g(r)$ indicates a partially ordered structure developing in the system at low densities which may be signaling a transition to the crystal phase. The interesting features seen in the zero and large frequency limits of $G(q, \omega)$ shed some light on the use of the local-field concept in 1D electron systems and may be important for further applications. Our findings may be tested experimentally as the quantum wire fabrication techniques improve. The Quantum Monte Carlo simulations and more refined theoretical approaches should also explore our predictions.

This work was partially supported by the Scientific and Technical Research Council of Turkey (TUBITAK). In the course of this work, CB was supported by a TUBITAK-NATO fellowship.

\section{REFERENCES}

[1] We give a few examples: Hill N. P. R., Nicholls J. T., Linfield E. H., Pepper M., Ritchie D. A., Jones G. A. C., Hu B. Y.-K. and Flensberg K., Phys. Rev. Lett., 78 (1997) 2204; Thomas K. J., Nicholls J. T., Simmons M. Y., Pepper M., Mace D. R. and Ritchie D. A., Phys. Rev. Lett., 77 (1996) 135; Schmeller A., Gõni A. R., Pinczuk A., Weiner J. S., Calleja J. M., Dennis B. S., Pfeiffer L. N. and West K. W., Phys. Rev. B, 49 (1994) 14778.

[2] Moudgil R. K., Ahluwalia P. K. and Pathak K. N., Phys. Rev. B, 52 (1995) 11945.

[3] Singwi K. S., Tosi M. P., Land R. H. and Suölander A., Phys. Rev., 179 (1968) 589; Singwi K. S. and Tosi M. P., Solid State Physics, 36 (1981) 177.

[4] Richardson C. F. and Ashcroft N. W., Phys. Rev. B, 50 (1994) 8170.

[5] Takayanagi K. and Lipparini E., Phys. Rev. B, 52 (1995) 1738; 54 (1996) 8122.

[6] Dobson J. F., Bünner M. J. and Gross E. K. U., Phys. Rev. Lett., 79 (1997) 1905. 
[7] Friesen W. I. and Bergersen B., J. Phys. C, 13 (1980) 6627; Borges A. N., Degani M. H. and Hipolito O., Superlatt. Microstruct., 13 (1993) 375; Gold A. and Calmels L., Solid State Commun., 100 (1996) 137.

[8] Calmels L. and Gold A., Phys. Rev. B, 52 (1995) 10841; Thakur J. S. and Neilson D., Phys. Rev. B, 56 (1997) 4679.

[9] Nafari N. and Davoudi B., Phys. Rev. B, 57 (1998) 2447; Calmels L. and Gold A., Phys. Rev. B, 57 (1998) 1436.

[10] Hasegawa T. and Shimuzu M., J. Phys. Soc. Jpn., 38 (1975) 965; Holas A. and Rahman S., Phys. Rev. B, 35 (1987) 2720; Schweng H. K. and Böнm H. M., Phys. Rev. B, 48 (1993) 2037.

[11] Hu G. Y. and O'Connell R. F., Phys. Rev. B, 42 (1990) 1290.

[12] Hu B. Y.-K. and Das Sarma S., Phys. Rev. B, 48 (1993) 5469.

[13] Ceperley D. M. and Alder B. J., Phys. Rev. Lett., 45 (1980) 566; Tanatar B. and Ceperley D. M., Phys. Rev. B, 39 (1989) 5005.

[14] Kalio A. and Pillo J., Phys. Rev. Lett., 77 (1996) 4237.

[15] Pederiva F., Lipparini E. and Takayanagi K., Europhys. Lett., 40 (1997) 607.

[16] Gasser W., Solid State Commun., 56 (1985) 121.

[17] Świerkowski L., Neilson D. and Szymański J., Phys. Rev. Lett., 67 (1991) 240.

[18] Moroni S. and Senatore G., Europhys. Lett., 16 (1991) 373; Likos C. N., Moroni S. and Senatore G., Phys. Rev. B, 55 (1997) 8867.

[19] Holas A., in Strongly Coupled Plasma Physics, edited by F. J. Rogers and H. E. DeWitT (Plenum, New York) 1987.

[20] Moroni S., Ceperley D. M. and Senatore G., Phys. Rev. Lett., 69 (1992) 1837; 75 (1995) 689. 